\title{
Non-infectious uveitis burden on quality of life and work impairment assessed through different psychometric questionnaires
}

European Journal of Ophthalmology I-9

(C) The Author(s) 2021 Article reuse guidelines: sagepub.com/journals-permissions DOI: $10.1177 / 11206721211034649$ journals.sagepub.com/home/ejo

\author{
Luca Cimino' (D), Piergiorgio Neri ${ }^{2,3}$ (D), Elisabetta Miserocchi ${ }^{4}$, \\ Maria Pia Paroli ${ }^{5}$, Lorenzo Vannozzi $i^{6}$, Leonardo Mastropasqua ${ }^{7}$, \\ Caterina Gagliano ${ }^{8}$, Maria Vadalà' (D), Michele Figus ${ }^{10}$ (D), \\ Francesca Romana Florio'", Barbara laccheri' ${ }^{12}$, Paolo Mora ${ }^{13}$, \\ Maurizio Fossarello ${ }^{14}$, Paola Balestrieri ${ }^{15}$, Giuliana Gualberti ${ }^{16}$, \\ Francesca Marando ${ }^{16}$ and Massimo Accorinti ${ }^{5}$
}

\begin{abstract}
Background: The purpose of this study was to evaluate the association between a novel psychometric 12-item questionnaire $(U$-qest) and other validated questionnaires to assess quality of life and work impairment in patients with non-infectious uveitis.

Methods: Data were collected at baseline and 3 months postbaseline using $U$-qest and two other validated questionnaires: The National Eye Institute 25-Item Visual Function Questionnaire (VFQ-25) and the 12-Item Short-Form Health Survey (SF-I2).

Results: A total of I 36 patients (52.2\% female) aged $47.9 \pm$ I 4.8 years (mean \pm SD) were enrolled in I 4 uveitis referral centres. U-qest correlated moderately with VFQ-25 and SF-12 at baseline and at 3 months. Both U-qest and VFQ-25 scores improved as disease improved; however, U-qest also detected improvement in patients for whom VFQ-25 scores did not improve. Disease activity was shown to significantly affect activity impairment. Patients and physicians expressed positive perceptions regarding the use and benefit of this instrument. U-qest showed very good reliability in terms of internal consistency (Cronbach's alpha $=0.91$ ).

Conclusions: U-qest can be considered a useful tool to assess the burden of uveitis on quality of life.
\end{abstract}

\section{Keywords}

Uveitis questionnaire, QoL, disease burden, work impairment

Date received: 9 February 202I; accepted: 28 June 2021

\footnotetext{
'Ocular Immunology Unit, Azienda USL-IRCCS, Reggio Emilia, Italy ${ }^{2}$ Cleveland Clinic Abu Dhabi, Abu Dhabi, United Arab Emirates

${ }^{3}$ Cleveland Clinic Lernear College of Medicine, Case Western Reserve University, Cleveland, OH, USA

${ }^{4}$ Department of Ophthalmology, San Raffaele Scientific Institute, VitaSalute University, Milan, Italy

${ }^{5}$ Uveitis Center, AOU Policlinico Umberto I, Sapienza University of Rome, Rome, Italy

${ }^{6}$ Department of Surgery and Translational Medicine, Eye Clinic, University of Florence, Florence, Italy

${ }^{7}$ Department of Medicine and Science of Ageing, Ophthalmology Clinic, University G. d'Annunzio Chieti-Pescara, Chieti, Italy

${ }^{8}$ Department of Ophthalmology, University Policlinic, Catania, Italy

${ }^{9}$ Department of Biomedicine, Neuroscience and Advanced Diagnostic, Ophthalmology Section, University of Palermo, Palermo, Italy
}

\footnotetext{
${ }^{10}$ Department of Surgical, Medical and Molecular Pathology and Critical Area Medicine, University of Pisa, Pisa, Italy

"Ospedale Monospecialistico Oftalmico, Rome, Italy

${ }^{12}$ Department of Biomedical and Surgical Sciences, Section of Ophthalmology, University of Perugia, S. Maria della Misericordia Hospital, Perugia, Italy

${ }^{13}$ Ophthalmology Unit, University Hospital of Parma, Parma, Italy

${ }^{14}$ Clinica Oculistica, Dipartimento di Scienze Chirurgiche, Università di

Cagliari, Cagliari, Italy

${ }^{15}$ Ospedale Antonio Cardarelli, Napoli, Italy

${ }^{16}$ Abbvie SRL, Rome, Italy

Corresponding author:

Luca Cimino, Ocular Immunology Unit, Azienda USL-IRCCS, Via

Risorgimento 80, 42I22 Reggio Emilia, Italy.

Email: cimino.luca@ausl.re.it
} 


\section{Introduction}

Uveitis represents a heterogeneous group of diseases affecting different portions of the uveal tract and other ocular tissues. ${ }^{1}$ It most frequently occurs in adults aged 20 50 years, ${ }^{2}$ affecting their quality of life (QoL) and carrying a social and economic burden. ${ }^{2}$ The QoL of patients with uveitis is affected not only by their visual impairment but also by associated systemic diseases and treatment-related side effects. ${ }^{3}$ Mental disorders have also been observed in patients affected by uveitis, negatively influencing work ability, study performance and career progression., ${ }^{3,4}$

Questionnaires have been developed to help measure health-related QoL in patients with vision-related disease, such as the National Eye Institute 25-Item Visual Function Questionnaire (VFQ-25) ${ }^{5}$ and Visual Function Questionnaire Utility Index. ${ }^{1}$ Both questionnaires evaluate patient perception on vision performance and provide a validated analysis of the data collected. However, the structure of these questionnaires is complex and could be problematic for patients with severe vision disability. ${ }^{3}$

The Medical Outcomes Study 36-Item Short Form Health Survey (SF-36), ${ }^{6,7}$ another validated questionnaire, was developed to provide eight dimensions. The 12-Item Short Form Health Survey (SF-12) is a shorter alternative to the SF-36. ${ }^{8}$

However, no studies on QoL and work productivity in patients affected by uveitis have been conducted in Italy thus far, perhaps owing to a lack of an appropriate QoL questionnaire. The aim of this study was to assess a new user-friendly e-questionnaire - U-qest - measuring QoL in patients affected by chronic or recurrent non-infectious uveitis.

\section{Methods}

\section{Patients and study design}

This prospective, multicentre, longitudinal, observational study compared the U-qest to the VFQ-25 and SF-12 in patients affected by chronic or recurrent non-infectious uveitis. It was conducted in 14 centres across Italy (Supplemental Table 1), with data collected at baseline, 2-7 days postbaseline (visit 2) and at 3 months postbaseline (visit 3).

Inclusion criteria were age $\geqslant 18$ years, a diagnosis of chronic or recurrent non-infectious uveitis, and the ability to understand and complete questionnaires. All questionnaires were completed by the patient in the presence of their physician. Patients excluded from the study were those affected by Fuchs uveitis, malignancies, human immunodeficiency virus or severe psychiatric comorbidities and those participating in other clinical trials on uveitis. The Standardization of Uveitis Nomenclature International Working Group ${ }^{9}$ criteria were used to assess uveitis status. Written informed consent was obtained from all patients and submitted to the local ethics committee in accordance with the Declaration of Helsinki.

\section{U-qest development, design and structure}

The U-qest was developed through three focus groups from July to November 2016 involving five Italian members of the International Uveitis Study Group treating physicians. The ophthalmologists, through a three-step process, drafted a set of dimensions and related questions (items) in Italian language, with the aim of evaluating the impact of uveitis on the QoL of patients. The questionnaire was designed to address the following 12 areas (Supplemental Table 2): general state of body self-perception, selfsufficiency, daily activities, work, psychological selfperception, mood, anxiety, personal relationships, social self-perception, future family plans, romantic relationships and social life. The questionnaire, written in Italian, was uploaded onto an electronic platform developed in accordance with the US Food and Drug Administration PRO Guidance for Industry. ${ }^{10}$ Patients completed the U-qest in the presence of a designated physician at baseline and at the two follow-up visits. Patients were asked to rank their answers using a visual analogue scale, with responses ranging from 0 ('absolutely not') to 10 ('definitely yes'). A smartphone application allowed patients to change the contrast and the size of the characters, read the questions aloud, confirm the chosen score and see a visual representation of the total score. Audio support was also included with this application. A graphical output was produced by linking the scores of the questions, facilitating comparison between visits (Supplemental Figure 1). The final score was the sum of the scores of all 12 questions, from 0 (no effect of the disease on QoL) to 120 (maximum effect of disease on QoL).

\section{VFQ-25, SF-I 2 and WPAI questionnaires}

The QoL questionnaires VFQ- $25^{5}$ and SF- $12^{8}$ as well as the Work Productivity and Activity Impairment (WPAI) ${ }^{11}$ questionnaire were used as control tests to validate U-qest at baseline and the two follow-up visits.

\section{Psychometric evaluation}

Psychometric evaluation was performed at different time points. Data collected at the first visit (baseline) were used to test the internal consistency and construct validity of the instrument; data collected at visit 2 were used to test the reliability of the instrument; data collected at 3 months (depending on routine visit schedules) were used to test the U-qest responsiveness to change.

\section{Construct validity}

To assess construct validity, the correlation of U-qest score versus VFQ-25 and SF-12 were analysed clinically and psychometrically at baseline. Differences in scores regarding severity of uveitis and QoL as measured by the VFQ25 and SF-12 were examined, hypothesizing that patients 
reporting higher levels of severity and lower QoL in those questionnaires should have higher U-qest scores.

\section{Responsiveness}

Responsiveness was tested using the paired $t$ test to compare the change in scores between two groups of patients between the first and last visit. Statistical significance was assessed both within groups (i.e. change in score among patients rating themselves as improved) and between groups (i.e. change in score among patients rating themselves as improved vs not improved). The change in clinical condition was tested in two different ways: based on the severity (active to inactive disease, according to physician's clinical evaluation) and based on the changes in activity of the disease, as perceived by the patient. Answers regarding patient satisfaction and perception ('According to you today the seriousness of your uveitis is. . ') were categorized into three classes ('very mild or mild'/'fair'/"high or very high') and patients rated as not improved or worsened are those whose perception of their severity of illness did not change or changed for the worse.

\section{Statistical analysis}

Test-retest reliability was estimated by administering the same questionnaire to the same patients at visit 2 . The Pearson's correlation coefficient between the two sets of responses was used as a quantitative measure of the testretest reliability.

Internal consistency reliability was determined using Cronbach's coefficient alpha to calculate the pairwise correlations between items. Internal consistency ranges from 0 to 1 . A commonly accepted rule of thumb is that an alpha of $0.6-0.7$ indicates acceptable reliability and $\geqslant 0.8$ indicates good reliability.

Sample size was determined according to feasibility criteria as previously described. ${ }^{12}$ According to these criteria, 10 subjects per questionnaire item were needed. The U-qest questionnaire comprises 12 items and, therefore, a minimum of 120 subjects was required. Data are presented using mean, standard deviation, median and range. Categorical variables are summarized by using frequency distribution and percentages. A $p$ value of $\leqslant 0.05$ was considered statistically significant. Statistical analyses were performed using Stata 13.0 (StataCorp LLC, College Station, TX, USA).

\section{Results}

\section{Patient baseline demographic characteristics}

A total of 136 patients were enrolled across 14 ophthalmology hospital/university centres. The mean \pm SD age was $47.9 \pm 14.8$ years, and $52.2 \%$ were female (Table 1 ). Most patients $(n=89[65 \%])$ had at least a high school degree, and most $(n=83[61 \%])$ were married or living
Table I. Demographics characteristics.

\begin{tabular}{|c|c|}
\hline General & $n=136$ \\
\hline Female sex, $n(\%)$ & $71(52.2)$ \\
\hline Age, mean $\pm S D$ (years) & $47.9 \pm 14.8$ \\
\hline $\mathrm{BMI}, \mathrm{kg} / \mathrm{m}^{2}$ & $25.1 \pm 4.2$ \\
\hline White race, $n(\%)$ & $130(95.6)$ \\
\hline \multicolumn{2}{|l|}{ Comorbid diseases, $n$ (\%) } \\
\hline$\geqslant$ I pathology & $55(40.4)$ \\
\hline Hypertension & $31(22.8)$ \\
\hline Obesity & $14(10.3)$ \\
\hline Dyslipidemia & $12(8.8)$ \\
\hline \multicolumn{2}{|l|}{ Medication, $n(\%)$} \\
\hline Corticosteroids, not systemic & $43(31.6)$ \\
\hline Topical & $40(29.4)$ \\
\hline Subtenon injection & $5(3.7)$ \\
\hline Intravitreal injection & $0(0.0)$ \\
\hline Intravitreal implant & I (0.74) \\
\hline Systemic corticosteroids & $51(37.5)$ \\
\hline Conventional immunosuppressive drugs & $4 \mid(30.2)$ \\
\hline Azathioprine & $13(9.6)$ \\
\hline Cyclosporin A & $15(\mid 1)$ \\
\hline Mycophenolate mofetil & $6(4.4)$ \\
\hline Methotrexate & $15(I I)$ \\
\hline Other & I (0.74) \\
\hline Biologic drugs & $30(22.1)$ \\
\hline
\end{tabular}

BMI: body mass index.

with a partner. Fifty-five patients $(40.4 \%)$ had $\geqslant 1$ comorbid disease, the most frequent being hypertension $(n=31$ [22.8\%]), and $51(37.5 \%)$ were receiving systemic corticosteroids. Other treatments for uveitis included systemic conventional immunosuppressive drugs, such as azathioprine $(n=13[9.6 \%])$, cyclosporin A $(n=15[11 \%])$, mycophenolate mofetil $(n=6[4.4 \%])$ or methotrexate $(n=15[11 \%])$. Thirty $(22.1 \%)$ patients were being treated with biological drugs.

\section{Uveitis disease characteristics}

Disease characteristics of patients with uveitis at study entry are summarized in Tables 2 and 3. Median (interquartile range) time from first symptoms of uveitis was 67 (32-123) months, and time from diagnosis was 55 (24116) months. The majority of patients $(n=89$ [65.4\%]) had uveitis in both eyes. Most patients had anterior uveitis $(n=51[37.5 \%])$ and panuveitis $(n=44[32.4 \%])$. The main cause of uveitis was idiopathic $(n=41$ [30.2\%]) or Behçet disease $(n=20[14.7 \%])$. One-third of patients had active uveitis $(n=45[33.1 \%])$.

\section{U-qest, VFQ-25, SF-I 2 and WPAl-uveitis questionnaires}

Although a slight reduction (improvement) in mean U-qest score versus baseline was observed in patients after 
Table 2. Disease characteristics.

\begin{tabular}{ll}
\hline Characteristic & $n=136$ \\
\hline Diagnosis, median (IQR), months & \\
Time from first symptoms of uveitis & $67(32.3-122.7)$ \\
Time from diagnosis of uveitis & $55(23.8-116.3)$ \\
Eyes affected, $n$ (\%) & \\
Right & $21(15.4)$ \\
Left & $26(19.1)$ \\
Both & $89(65.4)$ \\
Anatomical classification of uveitis, $n(\%)$ & \\
Anterior & $51(37.5)$ \\
Intermediate & $11(8.1)$ \\
Posterior & $30(22.1)$ \\
Panuveitis & $44(32.4)$ \\
Uveitis etiology, $n$ (\%) & \\
Idiopathic & $41(30.2)$ \\
Behçet disease & $20(14.7)$ \\
Other etiology & $15(11)$ \\
Ax-SpA & $14(10.3)$ \\
HLA-B27 related & $12(8.8)$ \\
Sarcoidosis & $8(5.9)$ \\
Vogt Koyanagi Harada & $7(5.2)$ \\
Complications of uveitis, $n$ (\%) & \\
Macular lesions & $20(14.7)$ \\
Synechiae & $18(13.2)$ \\
Cataract & $13(9.6)$ \\
Blindness & $6(4.4)$ \\
Glaucoma & $6(4.4)$ \\
Epiretinal membrane & $4(2.9)$ \\
Other & $9(6.6)$ \\
\hline
\end{tabular}

Ax-SpA: axial spondyloarthritis; IQR: interquartile range.

3 months, scores for other questionnaires remained largely unchanged between the two visits (Figure 1(a)). Stratifying patients by active versus inactive disease showed a similar trend among the different questionnaires at baseline and the 3-month visit (Figure 1(b) and (c)). At baseline, scores indicating a worse QoL were observed in patients with active versus inactive disease for U-qest, VFQ-25 and SF-12 Mental Component Summary (MCS; Figure 1(b)), and, although this trend was maintained at 3 months, only the VFQ-25 questionnaire detected a significant difference between groups (Figure 1(c)).

The effect of uveitis on a patient's ability to work and productivity was also assessed in patients with active versus inactive disease using the WPAI uveitis score (Figure 2). No difference was observed among the four measures (absenteeism, presenteeism, work impairment or activity impairment) between baseline and the 3-month visit (Figure 2(a)), whereas when stratifying patients by disease activity (inactive vs active disease), statistically significant differences in the burden of active disease on workrelated measures (with the exception of absenteeism)
Table 3. Clinical signs of uveitis at baseline.

\begin{tabular}{|c|c|c|}
\hline & \multicolumn{2}{|l|}{ Patients, $n=136$} \\
\hline & Left eye & Right eye \\
\hline \multicolumn{3}{|l|}{ Ophthalmic signs } \\
\hline BCVA, median (IQR) & $0.1(0-0.4)$ & $0.075(0-0.39)$ \\
\hline IOP, median (IQR) & $13(11.5-15)$ & $13(11-15)$ \\
\hline \multicolumn{3}{|c|}{ Clinical evaluation, $n(\%)$} \\
\hline Active & 45 (33.09)3.09) & \\
\hline Inactive & $91(66.91)$ & \\
\hline \multicolumn{3}{|l|}{ AC cells, $n(\%)$} \\
\hline 0 & 110 (80.88) & $112(82.35)$ \\
\hline+0.5 & II (8.09) & II (8.09) \\
\hline+1 & $10(7.35)$ & $10(7.35)$ \\
\hline+2 & $4(2.94)$ & $2(1.47)$ \\
\hline+3 & I (0.74) & $0(0.0)$ \\
\hline+4 & $0(0.0)$ & $0(0.0)$ \\
\hline NA & $0(0.0)$ & I (0.74) \\
\hline \multicolumn{3}{|l|}{ Flare, $n(\%)$} \\
\hline 0 & II 5 (84.56) & II 8 (86.76) \\
\hline+1 & $18(13.24)$ & $15(11.03)$ \\
\hline+2 & $3(2.2 I)$ & $2(1.47)$ \\
\hline+3 & $0(0.0)$ & $0(0.0)$ \\
\hline+4 & $0(0.0)$ & $0(0.0)$ \\
\hline NA & $0(0.0)$ & I (0.74) \\
\hline \multicolumn{3}{|l|}{ Vitreous haze, $n(\%)$} \\
\hline 0 & I I 6 (85.29) & I I 6 (85.29) \\
\hline+0.5 & 14 (10.29) & $9(6.62)$ \\
\hline+1 & $4(2.94)$ & $7(5.15)$ \\
\hline+2 & $2(1.47)$ & $3(2.21)$ \\
\hline+3 & $0(0.0)$ & $0(0.0)$ \\
\hline+4 & $0(0.0)$ & $0(0.0)$ \\
\hline NA & $0(0.0)$ & I (0.74) \\
\hline \multicolumn{3}{|c|}{ Cystoid macular oedema by OCT, $n$ (\%) } \\
\hline Presence & $20(|4.7|)$ & $15(11.03)$ \\
\hline Absence & $106(77.94)$ & III (81.62) \\
\hline NA & $10(7.35)$ & $10(7.35)$ \\
\hline
\end{tabular}

AC: anterior chamber; BCVA: best corrected visual acuity; IOP: intraocular pressure; IQR: interquartile range; NA: not available; OCT: optical coherence tomography.

were observed at both baseline (Figure 2(b)) and 3 months (Figure 2(c)).

When patients were stratified by the presence or absence of underlying systemic diseases, we observed that the SF-12 Physical Component Summary (PCS) was significantly worse (i.e. lower) at 3 months for those with $(46 \pm 8.7)$ versus without $(50.98 \pm 6.3)$ systemic disease $(p<0.001)$. No differences were observed at 3 months for the other questionnaires.

When patients were stratified according to anatomic classification, a similar trend was observed in the U-qest and VFQ-25 questionnaires at baseline (Supplemental Figure 2(a)) and 3 months (Supplemental Figure 2(b)): 


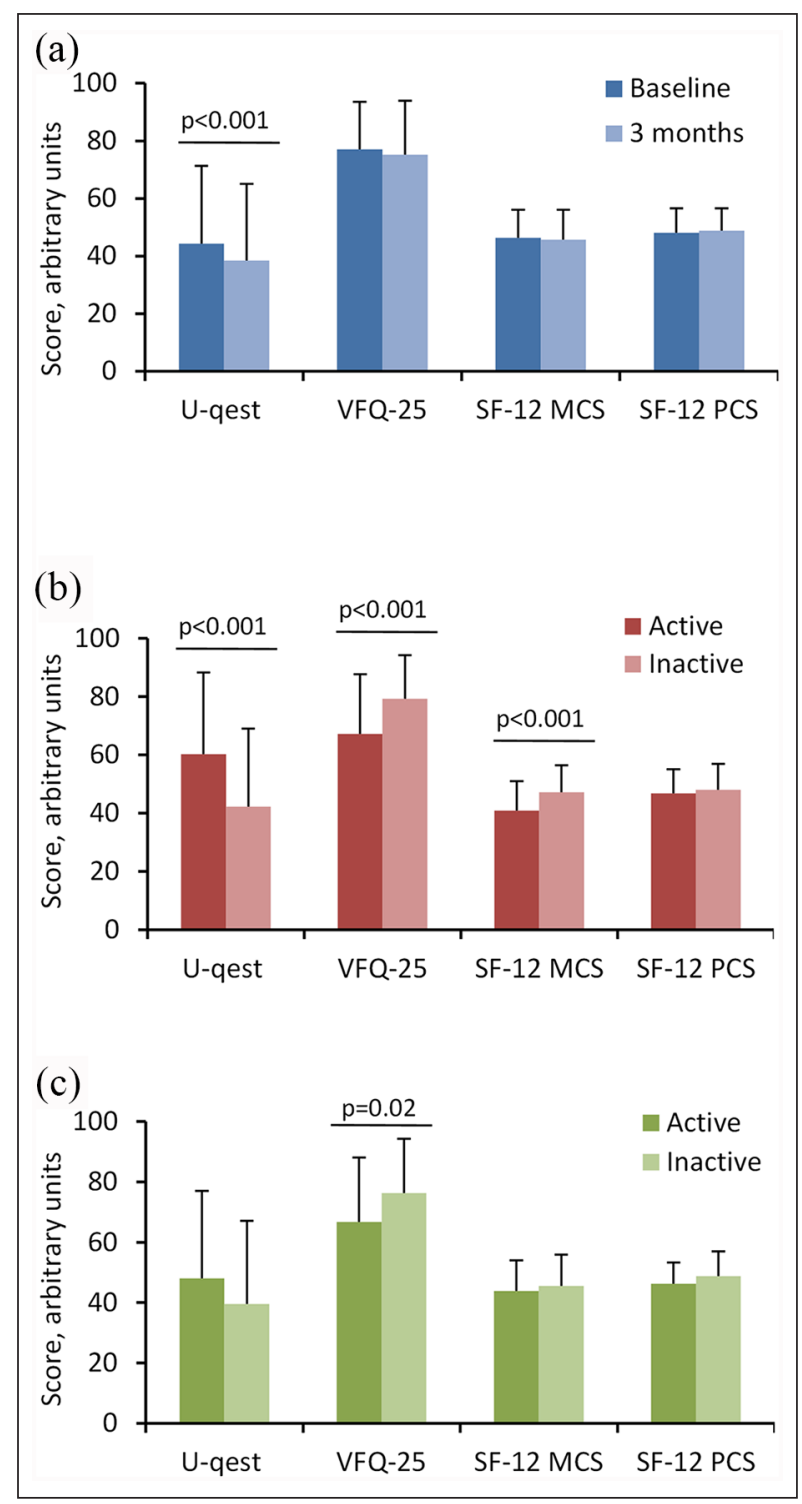

Figure I. U-qest, VFQ-25 and SF-12 questionnaires scores. Scores are presented as mean $\pm S D$, and $p$ values represent statistically significant differences between active and inactive disease groups based on all patients $(N=136)$. (a) Baseline and 3 months values. (b) Baseline active versus inactive values. (c) 3-month active versus inactive values. The VFQ-25 is composed of 25 items and was developed to assess the influence of visual impairment and visual symptoms in general health. ${ }^{5}$ It is used to assess the QoL in individuals with chronic eye diseases through 12 domains, I related to general health and II specifically related to vision. All subscale scores range from 0 to 100 , with 100 representing maximum visual function. The SF- 12 consists of 12 questions covering eight domains to measure health, including physical and mental components of QoL. ${ }^{8}$ The analysis of these areas results in two summary measures: Physical Component Summary (PCS) and the Mental Component Summary (MCS). Subscale scores range from $0 \%-100 \%$, with higher scores indicating better health.

VFQ-25: 25-Item Visual Function Questionnaire; SF-I2: I2-Item Short Form Health Survey; MCS: mental component summary; PCS: physical component summary.

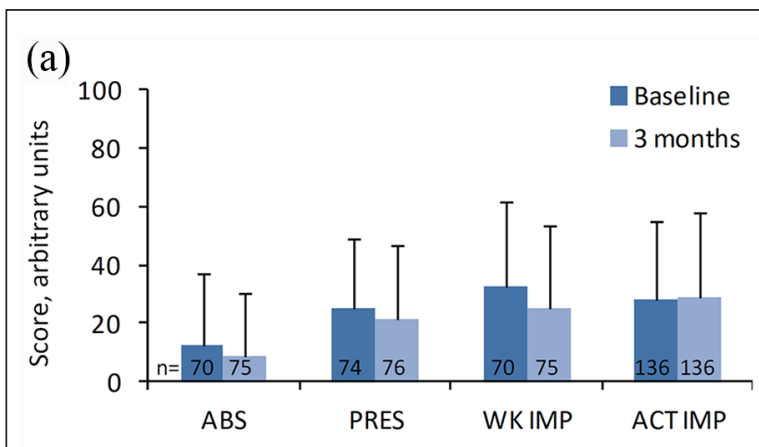

(b)

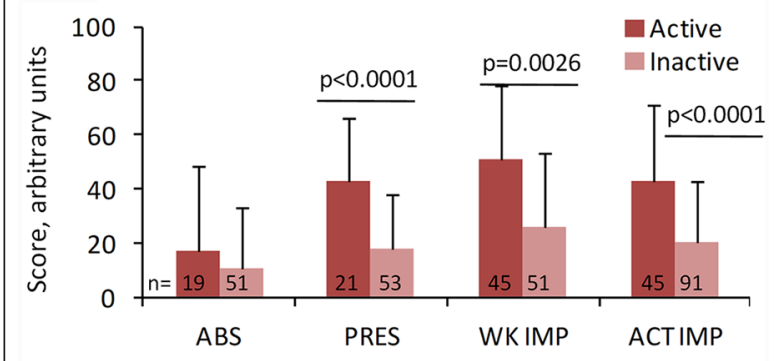

(c)

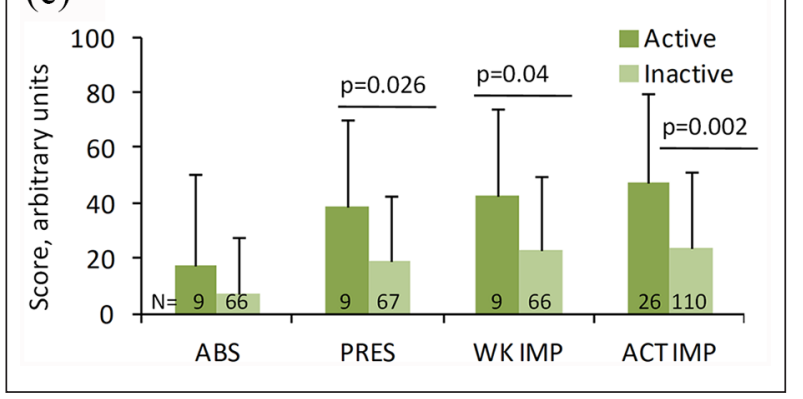

Figure 2. Effect of uveitis on the ability to work and on productivity using the WPAI uveitis questionnaire. Scores are presented as mean $\pm S D$, and $p$ values represent statistically significant differences between active and inactive disease groups. (a) Baseline and 3-month values. (B) baseline active versus inactive values. (C) 3-month active versus inactive values. The WPAl questionnaire was used to measure the effect of overall health and specific symptoms on productivity at work and outside work. " It consists of six items evaluating four domains: (I) activity impairment (percentage impairment in daily activities), (2) overall work productivity loss, (3) presenteeism (percentage of impairment experienced at work owing to health problems) and (4) absenteeism (percentage of working time missed owing to health problems). Higher scores indicate a greater degree of work productivity loss and activity impairment. ABS: absenteeism; ACT IMP: activity impairment; PRES: presenteeism; WK IMP: work impairment; WPAl: work productivity and activity impairment.

patients with intermediate uveitis reported less severe disease compared with those with panuveitis, who reported a higher burden of disease (Supplemental Figure 2(a) and 
Table 4. Patients' and physicians' perceptions of $U$-qest at baseline and at 3-month visit.

\begin{tabular}{|c|c|c|c|c|}
\hline & \multicolumn{2}{|c|}{ Patient's perception, $n(\%) n=136$} & \multicolumn{2}{|c|}{ Physician's perception, $n(\%) n=13$} \\
\hline & Baseline & 3 months & Baseline* & 3 months* \\
\hline \multicolumn{5}{|c|}{ Question I: According to you today the seriousness of your uveitis is. . . } \\
\hline Very mild & $8(5.88)$ & II (8.09) & & \\
\hline Mild & $28(20.59)$ & $30(22.06)$ & & \\
\hline Fair & $72(52.94)$ & $64(47.06)$ & & \\
\hline High & $25(18.38)$ & $27(19.85)$ & & \\
\hline Very high & $3(2.21)$ & $4(2.94)$ & & \\
\hline \multicolumn{5}{|c|}{ Question 2: Are you satisfied with the use of the $U$-qest? } \\
\hline Not at all & I (0.74) & $0(0.0)$ & $0(0.0)$ & $0(0.0)$ \\
\hline Just a little & $4(2.94)$ & $5(3.68)$ & $0(0.0)$ & $0(0.0)$ \\
\hline Moderately & $14(10.29)$ & 27 (19.85) & I (7.69) & I (7.69) \\
\hline Quite a lot & 70 (5 I.47) & $68(50.00)$ & $4(30.77)$ & $4(30.77)$ \\
\hline Very much so & $47(34.56)$ & $36(26.47)$ & $8(6 \mid .54)$ & $8(61.54)$ \\
\hline \multicolumn{5}{|c|}{$\begin{array}{l}\text { Question 3: Have the } 12 \text { questions from the } U \text {-qest been enough to provide your physician with a satisfactory overall understanding } \\
\text { of the problems you experience because of your uveitis? }\end{array}$} \\
\hline Not at all & $4(2.94)$ & $0(0.0)$ & $0(0.0)$ & $0(0.0)$ \\
\hline Just a little & $9(6.62)$ & 14 (10.29) & $0(0.0)$ & $0(0.0)$ \\
\hline Moderately & 21 (15.44) & $29(21.32)$ & $2(15.38)$ & $2(15.38)$ \\
\hline Quite a lot & $75(55.15)$ & $67(49.26)$ & $6(46.15)$ & $4(30.77)$ \\
\hline Very much so & $27(19.85)$ & $26(19.12)$ & $5(38.46)$ & $7(53.85)$ \\
\hline \multicolumn{5}{|c|}{ Question 4: Has the use of the $U$-qest improved the relationship with your ophthalmologist for uveitis? } \\
\hline Not at all & $6(4.4 I)$ & $3(2.21)$ & & \\
\hline Just a little & $12(8.82)$ & $16(11.76)$ & & \\
\hline Moderately & $21(15.44)$ & $29(21.32)$ & & \\
\hline Quite a lot & $63(46.32)$ & $55(40.44)$ & & \\
\hline Very much so & $34(25.00)$ & $33(24.26)$ & & \\
\hline
\end{tabular}

*Missing data; one physician was not available to provide data.

(b)). No difference based on anatomical classification was observed at baseline or 3 months according to SF-12 MCS or PCS.

\section{Patient and physician perception of U-qest questionnaire}

Patient perception of the U-qest questionnaire was assessed through four specific questions (Table 4). Approximately $70 \%$ of patients reported that the severity of their uveitis was between 'fair' and 'very high' (question 1), and approximately $90 \%$ of patients reported overall positive perception ('moderately', 'quite a lot' and 'very much') when assessing this instrument (questions 2 and 3) and its role in improving their relationship with their ophthalmologist (question 4). No discernible difference was observed in patient perception of the questionnaire from baseline to the 3-month visit (Table 4). Physician perception of the U-qest was also very positive and higher than patient perceptions; $61.54 \%$ of physicians reported that they were 'very much' satisfied with the questionnaire, and an increased percentage of physicians (from 38.46\% at baseline to $53.85 \%$ at 3 months) reported being 'very much' satisfied with this questionnaire for providing them with a better understanding of the problems experienced by their patients owing to uveitis.

\section{Construct validity}

The grade of correlation of the U-qest score with the VFQ25 was moderate $(-0.60$ [95\% CI, -0.72 to -0.49$])$. The grade of correlation of the U-qest score with the SF-12 was low for PCS $(-0.37$ [95\% CI, -0.52 to -0.23$])$ and moderate for MCS $(-0.55[95 \% \mathrm{CI},-0.67$ to -0.43$])$.

\section{Internal consistency and stability}

The overall reliability of the U-qest questionnaire was very good for both internal consistency (Cronbach's alpha, 0.91) and stability: according to test-retest, the Pearson correlation with baseline for the second visit was 0.75 (95\% CI, 0.61 to 0.89 ).

\section{Responsiveness}

The change in questionnaire scores (in terms of activity and severity of disease) from baseline to 3 months was evaluated from the perspective of the physician and 
patient (Supplemental Table 3). After 3 months, patients who improved and patients who did not improve or who remained unchanged (according to their physicians) showed a significant decrease in U-qest score compared with baseline; U-qest reports a statistically significant improvement in QoL, although to a different extent. There was no significant difference between patients who improved, and those who did not improve or remained unchanged.

Regarding VFQ-25, patients who improved, on average, showed a score increase, although it was not statistically significant. Patients who did not improve or worsened had a significant mean score decrease, therefore VFQ-25 scores moved in the expected direction. When comparing these mean changes between groups we observed a significant difference.

For SF-12 PCS and MCS, both groups of patients reported no change, on average and no significant difference between groups was observed. When this analysis was repeated to evaluate U-qest score according to question 1 of the patients' perception of the severity of illness (activity of disease), results were similar to those observed for physicians' perception.

\section{Discussion}

Current literature on the effect of chronic or recurrent non-infectious uveitis on QoL in the Italian population is largely limited, with no studies available on its effect on work impairment or productivity. Two Italian studies were previously conducted, ${ }^{6,13}$ but both were single-centre studies and both used the SF-36 questionnaire, which does not collect information on all the areas of well-being and functioning that may be of importance to patients with uveitis. These studies ${ }^{6,13}$ and others not performed in Italy ${ }^{14-16}$ have consistently shown that QoL is significantly affected in patients with non-infectious uveitis compared with healthy individuals.

To address this unmet need, the U-qest questionnaire was developed to explore 12 general areas reflecting QoL. In fact, whereas the VFQ-25 evaluates vision-specific aspects and the SF-12 is a generic measure of healthrelated QoL, U-qest was designed as a generic measure of those aspects of QoL specifically affected by uveitis.

Several important findings have emerged from the present study. We observed a moderate correlation between U-qest scores and VFQ-25 and SF-12 questionnaire scores at baseline. In patients with an improvement in disease, both U-qest and VFQ-25 scores improved, however, U-qest also detected a significant improvement in patients for whom VFQ-25 scores reported stable or worsening disease. This may be attributed to the low sample size, other comorbid diseases in this subgroup or heterogeneity of the aetiology and the variability of the clinical picture of uveitis. It is also possible that the study duration was insufficient to detect a measurable improvement, or that the patient's positive reaction to the attention paid by ophthalmologists to their QoL and well-being affected their scores.

Furthermore, we observed that work and productivity impairment were significantly affected in patients with active versus inactive disease using the WPAI uveitis questionnaire, confirming previous findings. ${ }^{17}$

Recent evidence from a study conducted in Spain showed that lower best corrected visual acuity and the presence of ocular comorbidities were negatively associated with vision-related QoL in patients with non-infectious uveitis. ${ }^{18}$ A study performed in Australia demonstrated that patients with uveitis had significantly poorer visionrelated and health-related QoL compared with healthy individuals. ${ }^{15}$ Although we did not observe any discernible differences in scores for patients with versus without ocular comorbidities among the different questionnaires (possibly because of the limited number of patients available), we did observe a significant worsening at 3 months in SF-12 PCS scores from baseline in patients with versus without systemic diseases.

No difference in questionnaire scores by classification of uveitis was observed, although we did observe a trend in which patients with posterior uveitis or panuveitis had more severe disease compared with anterior uveitis. This trend was seen in U-qest and VFQ-25 questionnaires only and corroborates with findings by Schiffman et al., ${ }^{14}$ but not with findings by Fabiani et al, in which a more severe effect on QoL in patients with acute anterior uveitis compared with those with panuveitis was found. ${ }^{13}$

Panuveitis is treated with systemic (e.g. corticosteroids and biologic agents) and topical therapies whereas anterior uveitis is usually managed with topical therapies only. Differences in underlying severity among patient cohorts as well as their therapeutic management may account for the differences observed across studies. ${ }^{13,14}$

Finally, we also evaluated the patient and physician perception of the questionnaire and observed favourable responses ( $>70 \%$ positive response). This feature of the questionnaire highlights the importance of the psychological burden of uveitis, often reflected in poor scores in the MCS in the SF-12 and SF-36 questionnaires, ${ }^{14-16}$ but not in all studies. ${ }^{13}$

Potential differences in degree of visual impairment may have influenced the capacity of some patients to complete the questionnaire, although this questionnaire was administered through an App that also provided audio output for each of the questions. The presence of a physician in these cases allows all patients the possibility to complete each questionnaire as accurately as possibly without the risk of incorrect completion or missing information. Moreover, the physician did not influence in any way the response given by the patient, to avoid any potential bias. In addition, one of the important characteristics of this 
study design was to share the experience in terms of monitoring the change and improvement in the patient, facilitating communication between the patient and physician and real-time visualization of their progress.

Although an assessment of clinical severity is always performed, the effect of non-infectious uveitis on the patient QoL is rarely performed outside specialized centres. Hence, patients are seldom asked to discuss the outcome of the tests in subsequent interviews. However, there is evidence that incorporating an instrument to assess the effect of a disease on the patient's QoL into the routine medical practice can induce greater patient satisfaction, improved compliance and even improve clinical outcome. ${ }^{19}$

The mean \pm SD age of patients who participated the present study $(47.9 \pm 14.9$ years $)$ was similar to other studies, ${ }^{14,20-22}$ highlighting that the visual impairment secondary to uveitis usually affects an adult population of working age, imposing changes in QoL with significant social and economic impact. Although it is recognized that age can impact upon QoL in uveitis patients using other questionnaires ${ }^{23}$ due to unequal distribution of age and low sample size, we did not stratify patients to assess this in further detail.

The assessment of disease severity and QoL in patients with non-infectious uveitis is not sufficiently performed in daily practice. This is mainly due to the lack of fast and visually sensitive instruments and intuitive tools, particularly regarding QoL.

Our tool is intended to be administered through a smartphone application, which would allow both physician and patient to have an immediate representation of the progress achieved. Having a global picture of the patient's status can help the clinician make decisions regarding therapy, even when uveitis is under control but the perceived burden of the current treatment is destabilizing for the patient.

\section{Conclusion}

Evaluating changes in QoL associated with the clinical course of disease is essential to improve the management of non-infectious uveitis. U-qest can be considered a useful tool for a global assessment of the effect of uveitis on QoL. This psychometric questionnaire provides an additional, disease-specific, useful, self-administered instrument that measures QoL, helping to develop a good patient-physician relationship. Future studies will aid in our understanding of the effect of non-infectious uveitis on QoL in relation to clinical course.

\section{Acknowledgements}

Medical writing support and editorial assistance was provided by Colin Gerard Egan, PhD (CE Medical Writing, Pisa, Italy), and was funded by AbbVie (no grant number). The authors thank Cesare Mariotti (Department of Ophthalmology, University of Ancona, Ancona, Italy) for his help in recruiting the patients and
Claudia Cornacchia of AbbVie, for U-qest realization and study implementation and management.

\section{Author contributions}

Luca Cimino, Piergiorgio Neri, Elisabetta Miserocchi, Maria Pia Paroli, Giuliana Gualberti and Massimo Accorinti contributed to the U-qest ideation and development and to protocol ideation. Francesca Marando, Giuliana Gualberti and Massimo Accorinti contributed to data analysis and interpretation of results. Luca Cimino, Elisabetta Miserocchi, Maria Pia Paroli, Lorenzo Vannozzi, Leonardo Mastropasqua, Caterina Gagliano, Maria Vadalà, Michele Figus, Francesca Romana Florio, Barbara Iaccheri, Paolo Mora, Maurizio Fossarello, Paola Balestrieri and Massimo Accorinti enrolled patients and collected study data. All authors equally contributed to the writing and reviewing of the manuscript and approved the final version.

\section{Declaration of conflicting interests}

The author(s) declared the following potential conflicts of interest with respect to the research, authorship, and/or publication of this article: G Gualberti and F Marando are AbbVie employees and may own AbbVie stocks and options. L Cimino received consultancy fees from AbbVie and Santen. All other authors have no conflict of interest to declare.

\section{Funding}

The author(s) disclosed receipt of the following financial support for the research, authorship, and/or publication of this article: AbbVie Srl (Rome, Italy) sponsored this study and editorial assistance for the writing of the manuscript. AbbVie participated in the study design, interpretation of data and writing of the publication.

\section{ORCID iDs}

Luca Cimino (iD https://orcid.org/0000-0001-7956-9950

Piergiorgio Neri (iD https://orcid.org/0000-0002-7673-4276

Maria Vadalà (iD https://orcid.org/0000-0002-2726-698X

Michele Figus (iD https://orcid.org/0000-0003-2243-9033

Maurizio Fossarello iD https://orcid.org/0000-0003-1520-7760

\section{Supplemental material}

Supplemental material for this article is available online.

\section{References}

1. Naik RK, Gries KS, Rentz AM, et al. Psychometric evaluation of the National Eye Institute Visual Function Questionnaire and Visual Function Questionnaire Utility Index in patients with non-infectious intermediate and posterior uveitis. Qual Life Res 2013; 22(10): 2801-2808.

2. Durrani OM, Tehrani NN, Marr JE, et al. Degree, duration, and causes of visual loss in uveitis. Br J Ophthalmol 2004; 88(9): 1159-1162.

3. De Smet MD, Taylor SRJ, Bodaghi B, et al. Understanding uveitis: the impact of research on visual outcomes. Prog Retin Eye Res 2011; 30(6): 452-470. 
4. Onal S, Oray M, Yasa C, et al. Screening for depression and anxiety in patients with active uveitis. Ocul Immunol Inflamm 2018; 26(7): 1078-1093.

5. Rentz AM, Kowalski JW, Walt JG, et al. Development of a preference-based index from the National Eye Institute Visual Function Questionnaire-25. JAMA Ophthalmol 2014; 132(3): 310-318.

6. Miserocchi E, Modorati G, Mosconi P, et al. Quality of life in patients with uveitis on chronic systemic immunosuppressive treatment. Ocul Immunol Inflamm 2010; 18(4): 297-304.

7. Patrick DL and Deyo RA. Generic and disease-specific measures in assessing health status and quality of life. Med Care 1989; 27(3 Suppl): S217-S232.

8. Ware J, Kosinski M and Keller SD. A 12-Item Short-Form Health Survey: construction of scales and preliminary tests of reliability and validity. Med Care 1996; 34(3): 220-233.

9. Jabs DA, Nussenblatt RB, and Rosenbaum JT; Standardization of Uveitis Nomenclature (SUN) Working Group. Standardization of uveitis nomenclature for reporting clinical data. Results of the First International Workshop. Am J Ophthalmol 2005; 140(3): 509-516.

10. Food and Drug Administration Guidance for Industry. Patient-reported outcome measures: use in medical product development to support labeling claims. https://www.fda. gov/regulatory-information/search-fda-guidance-documents/ patient-reported-outcome-measures-use-medical-productdevelopment-support-labeling-claims (December 2009).

11. Reilly MC, Gooch KL, Wong RL, et al. Validity, reliability and responsiveness of the Work Productivity and Activity Impairment Questionnaire in ankylosing spondylitis. Rheumatology (Oxford) 2010; 49(4): 812-819.

12. Streiner DL, Norman GR and Cairney J. Health measurement scales: a practical guide to their development and use. 5th ed. Oxford: Oxford University Press, 2014.

13. Fabiani C, Vitale A, Orlando I, et al. Impact of uveitis on quality of life: a prospective study from a tertiary referral
Rheumatology-Ophthalmology Collaborative Uveitis Center in Italy. Isr Med Assoc J 2017; 19(8): 478-483.

14. Schiffman RM, Jacobsen G and Whitcup SM. Visual functioning and general health status in patients with uveitis. Arch Ophthalmol 2001; 119(6): 841-849.

15. Hui MM, Wakefield D, Patel I, et al. Visual functioning and health-related quality-of-life are compromised in patients with uveitis. Ocul Immunol Inflamm 2017; 25(4): 486-491.

16. Maca SM, Wagner J, Weingessel B, et al. Acute anterior uveitis is associated with depression and reduction of general health. Br J Ophthalmol 2013; 97(3): 333-337.

17. Jalil A, Yin K, Coyle L, et al. Vision-related quality of life and employment status in patients with uveitis of working age: a prospective study. Ocul Immunol Inflamm 2012; 20(4): 262-265.

18. Arriola-Villalobos P, Abásolo L, García-Feijoo J, et al. Vision-related quality of life in patients with non-infectious uveitis: a cross-sectional study. Ocul Immunol Inflamm 2018; 26(5): 717-725.

19. Linder D, Sampogna F, Torreggiani A, et al. Psodisk, a new visual method for assessing the burden of psoriasis on patients. J Eur Acad Dermatol Venereol 2012; 26(9): 1163-1166.

20. Rothova A, Suttorp-van Schulten MS, Frits Treffers W, et al. Causes and frequency of blindness in patients with intraocular inflammatory disease. Br J Ophthalmol 1996; 80(4): 332-336.

21. Durrani OM, Meads CA and Murray PI. Uveitis: a potentially blinding disease. Ophthalmologica 2004; 218(4): 223-236.

22. Frick KD, Drye LT, Kempen JH, et al. Associations among visual acuity and vision- and health-related quality of life among patients in the multicenter uveitis steroid treatment trial. Invest Ophthalmol Vis Sci 2012; 53(3): 1169-1176.

23. Grajewski RS, Boelke AC, Adler W, et al. Perceived stress levels in adult patients with uveitis. Front Psychiatry 2020; 10: 916 . 